\title{
Avaliação do desempenho de diferentes classificadores (Isoseg, Bhattacharyya, Maxver e Maxver-ICM), utilizando imagens \\ CCD/CBERS-1 e ETM ${ }^{+} /$Landsat-7 fusionadas
} (doi:10.4136/ambi-agua.14)

\author{
Camila Aguirre Góes ${ }^{1}$; Wilson Lins de Mello Filho²; Melissa Carvalho ${ }^{3}$ \\ ${ }^{1}$ Instituto Nacional de Pesquisas Espaciais (INPE), Divisão de Sensoriamento Remoto. Av. dos \\ Astronautas, 1758. Cep: 12.227-010. São José dos Campos/SP, Brasil. E-mail: camila@dsr.inpe.br. \\ ${ }^{2}$ Instituto de Estudos do Mar Almirante Paulo Moreira. Rua Kioto, 253. CEP: 23.890-000. Arraial do \\ Cabo/RJ, Brasil. E-mail: linsdemello@ieapm.mar.mil.br. \\ ${ }^{3}$ Instituto Oceanográfico, Universidade de São Paulo. Praça do Oceanográfico, 191. CEP: 05508-900. São \\ Paulo/SP, Brasil. E-mail: melissa@io.usp.br
}

\section{RESUMO}

O objetivo deste estudo é comparar o desempenho de classificadores de imagens (Isoseg, Bhattacharyya, Maxver e Maxver-ICM) a partir da análise de acurácia (porcentagem de acertos, cálculo de área e estatística Kappa), utilizando como verdade terrestre um mapa temático previamente editado. Para alcançar este objetivo foram utilizadas técnicas de préprocessamento (correções atmosférica, geométrica e radiométrica), realce (fusão IHS e análise por componentes principais) e classificação das imagens CCD/CBERS-1 e $\mathrm{ETM}^{+} /$Landsat-7. Dentre os classificadores testados, o Isoseg e o Bhattacharyya apresentaram melhor desempenho para a área de estudo e para as classes representadas. Espera-se que esses resultados sejam relevantes para a análise ambiental com base em imagens orbitais.

Palavras-chave: fusão IHS; coeficiente Kappa; classificação.

\section{Performance evaluation of different classifiers (Isoseg, Bhattacharyya, Maxver e Maxver-ICM), using CCD/CBERS-1 and ETM ${ }^{+} /$LANDSAT- 7 fused images}

\begin{abstract}
The aim of this study was to compare the performance of image classifiers (Isoseg, Bhattacharyya, Maxver and Maxver-ICM) based on an accuracy analysis (set percentage, area determination and Kappa coefficient), using as ground truth an edited thematic map. For this, pre-processing techniques (atmospheric, geometric and radiometric corrections), contrast enhancement (IHS data fusion and principal component analysis) and classification of CCD/CBERS-1 and ETM ${ }^{+} /$Landsat-7 images were done. Amongst all classifiers tested, Isoseg and Bhattacharyya presented best performance for the studied classes and the study area. It is anticipated that these results are relevant to environmental analyses based on orbital satellite data.
\end{abstract}

Keywords: IHS fusion; Kappa coefficient; classification. 


\section{INTRODUÇÃO}

O sensoriamento remoto orbital tem fornecido informações acerca dos processos naturais e dos impactos antrópicos sobre o meio ambiente, proporcionando uma nova dimensão para o entendimento destes processos. Os dados obtidos por plataformas orbitais apresentam um enorme potencial de utilização para o manejo e monitoramento global (Barrett; Curtis, 1992). Para que os dados gerados por sensoriamento remoto sejam utilizados corretamente e com o máximo de aproveitamento, faz-se necessária a aplicação de um conjunto de ferramentas matemáticas e estatísticas digitais que possibilitem a observação e a garantia da correção das informações contidas nos seus produtos finais. As técnicas de Processamento Digital de Imagens (PDI) existentes se propõem a suprir essa necessidade e são aplicadas em duas etapas: pré-processamento e processamento da imagem. A primeira objetiva preparar os dados adquiridos pelo sensor para serem corretamente utilizados, empregando-se técnicas para correção atmosférica e correção de distorções radiométricas e geométricas introduzidas pelo sensor. Na etapa seguinte, são efetuados procedimentos com o objetivo de, efetivamente, extrair informações da imagem. Maiores detalhes sobre as etapas apresentadas podem ser encontrados em Lillesand et al. (2004), Mather (2004), Richards e Jia (2006), entre outros.

No presente estudo foram utilizadas técnicas de pré-processamento e processamento em imagens CCD/CBERS-1 e ETM ${ }^{+} /$Landsat-7, com o objetivo de avaliar o desempenho de quatro classificadores (Isoseg, Bhattacharyya, Maxver, Maxver-ICM), analisando a acurácia da classificação a partir da porcentagem de acertos (matriz de erro), do cálculo de área e do coeficiente Kappa.

\section{MATERIAL E MÉTODOS}

\section{1. Área de estudo}

A área de estudo se estende por 261,35 km² dentro do estado do Ceará, entre as latitudes $4^{\circ} 31^{\prime} \mathrm{S}$ e $4^{\circ} 38^{\prime} \mathrm{S}$ e longitudes $37^{\circ} 48^{\prime} \mathrm{W}$ e $37^{\circ} 38^{\prime} \mathrm{W}$ (Figura 4) e está inserida na bacia sedimentar Potiguar. A área escolhida apresenta uma grande diversidade de classes com comportamento espectral diferenciado, sendo apropriada para avaliar o desempenho dos classificadores em relação a cada classe. Neste estudo foram definidas seis classes temáticas, sendo três diferentes tipos de vegetação (Vegetação 1, Vegetação 2 e Manguezal), Água (incluindo lagoas, rios e oceano), Duna e Área Urbana.

\subsection{Pré-processamento}

Foram utilizadas imagens provenientes de dois sensores, CCD e $\mathrm{ETM}^{+}$, a bordo dos satélites CBERS-1 e Landsat-7, respectivamente. Da imagem ETM $^{+}$utilizou-se apenas a banda pancromática $(0,52-0,90 \mu \mathrm{m})$, pré-processada por Carvalho (2003) e as bandas $2(0,52-$ 0,59 $\mu \mathrm{m}), 3(0,63-0,69 \mu \mathrm{m})$ e $4(0,77-0,89 \mu \mathrm{m})$ da imagem CCD. As imagens foram processadas de acordo com os procedimentos resumidos na Figura 1, utilizando-se os programas Restau - Image Restoration 0.0.15 (Fedorov; Fonseca, 2002), SPRING 4.1 (Camara et al., 1996) e Idrisi Kilimanjaro.

Na imagem CCD, procedeu-se à correção atmosférica pelo método proposto por Chavez (1988). Posteriormente, foi realizado o ajuste de média e variância das bandas 2 e 3 a partir da banda 4. Finalmente, foi realizada a correção radiométrica e reamostragem dos pixels de 20 para 10 metros, utilizando-se o programa Restau. A banda pancromática (PAN) do sensor $\mathrm{ETM}^{+} /$Landsat-7 foi previamente reamostrada de 15 para 5 metros, utilizando-se o mesmo programa Restau. Ao término dessa etapa, procedeu-se à correção geométrica, em que foi 
efetuado o registro das bandas 2, 3 e 4 do CCD/CBERS-1 a partir da banda pancromática $\mathrm{ETM}^{+} /$Landsat-7. Dessa forma, permitiu-se que a exatidão cartográfica fosse mantida segundo o sistema de coordenadas UTM/SAD 69.

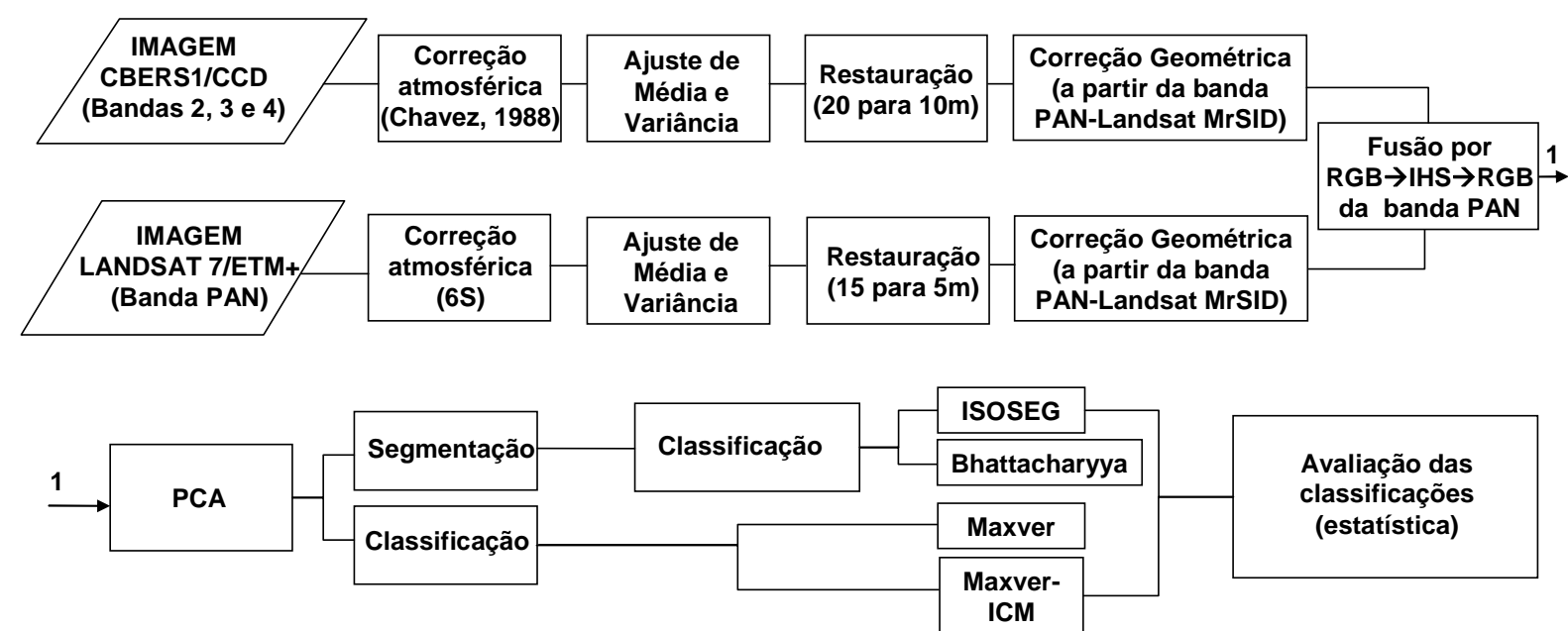

Figura 1. Seqüência metodológica utilizada para o processamento das duas imagens usadas no trabalho (CCD/CBERS-1 e ETM+/Landsat).

\subsection{Realce de imagem}

Após o pré-processamento foi realizada a fusão por transformação IHS, em que o componente Intensity (I) do sistema IHS foi substituído pela banda PAN (ETM ${ }^{+} /$Landsat-7). Desta forma, obteve-se uma imagem com resolução espacial de cinco metros com características espectrais originais.

$\mathrm{Na}$ imagem fusionada, aplicou-se a transformação por Componentes Principais. Essa técnica é utilizada para descorrelacionar as bandas espectrais, concentrando no Primeiro Componente (PC-1) a informação anteriormente diluída nas várias dimensões. Esse componente passa a reunir a informação de brilho associada às sombras de topografia e às grandes variações de reflectância espectral geral das três bandas e foi utilizada para ser segmentada e classificada.

\subsection{Classificação de imagem}

A imagem resultante do primeiro Componente Principal foi realçada e segmentada pela técnica de crescimento de regiões e, posteriormente, classificada a partir dos algoritmos Isoseg e Bhattacharyya. O primeiro algoritmo agrupa regiões a partir de uma medida de similaridade (distância de Mahalanobis), enquanto que o método Bhattacharyya trabalha com a distância denominada distância de Bhattacharyya, que é utilizada para medir a separabilidade estatística entre um par de classes espectrais, ou seja, mede a distância média entre as distribuições de probabilidades dessas classes (INPE, 2004; Mather, 2004). Dentre os limiares de segmentação testados o que se mostrou mais adequado para a área de estudo foi o de $95 \%$.

A mesma imagem do primeiro Componente Principal foi utilizada para classificação "pixel a pixel” utilizando os algoritmos Maxver (Máxima Verossimilhança) e Maxver- ICM (Interated Conditional Modes). O algoritmo Maxver considera a ponderação das distâncias entre médias dos níveis digitais das classes, utilizando parâmetros estatísticos na classificação, já o algoritmo utilizado pelo classificador Maxver-ICM funciona de forma semelhante ao do Maxver, porém considera ainda a dependência espacial entre as classes (INPE, 2004; Mather, 2004). 


\subsection{Análise comparativa dos produtos}

Como não se dispunha de amostras coletadas em campo para validar o experimento, utilizou-se como verdade de campo o mapa de uso e cobertura do solo, produzido por Carvalho (2003). Esse mapa continha um número de classes maior do que as definidas neste estudo, sendo necessário o remapeamento das classes para efeitos comparativos.

A verificação da acurácia dos classificadores foi realizada a partir da porcentagem de acertos (matriz de erro) (Congalton et al., 1983), que expressa a concordância e discordância entre as categorias classificadas em um mapa de uso e cobertura do solo e a verdade terrestre (Rosenfield, 1986; Rosenfield; Fitzpatrick-Lins, 1986), do cálculo de área (em $\left.\mathrm{km}^{2}\right)$ e da estatística Kappa, utilizada por vários autores para avaliar a concordância entre a verdade terrestre e os resultados obtidos a partir das classificações (Cohen, 1960; Rosenfield; Fitzpatrick-Lins, 1986; Foody, 1992).

\section{RESULTADOS E DISCUSSÃO}

\subsection{Pré-processamento}

Cada procedimento de pré-processamento utilizado foi responsável por melhorar algum aspecto da imagem. O pré-processamento constou de correção atmosférica, promovendo uma maior nitidez dos alvos (Figura 2B). Posteriormente, foi realizado o ajuste de média e de variância das bandas 2 e 3 a partir da banda 4 (banda que retém maior quantidade de informação), proporcionando um melhor contraste da imagem (Figura 2C). E, finalmente, a reamostragem dos pixels de 20 para 10 metros, promovendo uma melhora da resolução espacial e recuperando a imagem degradada pela resolução efetiva do sensor (Figura 2D).
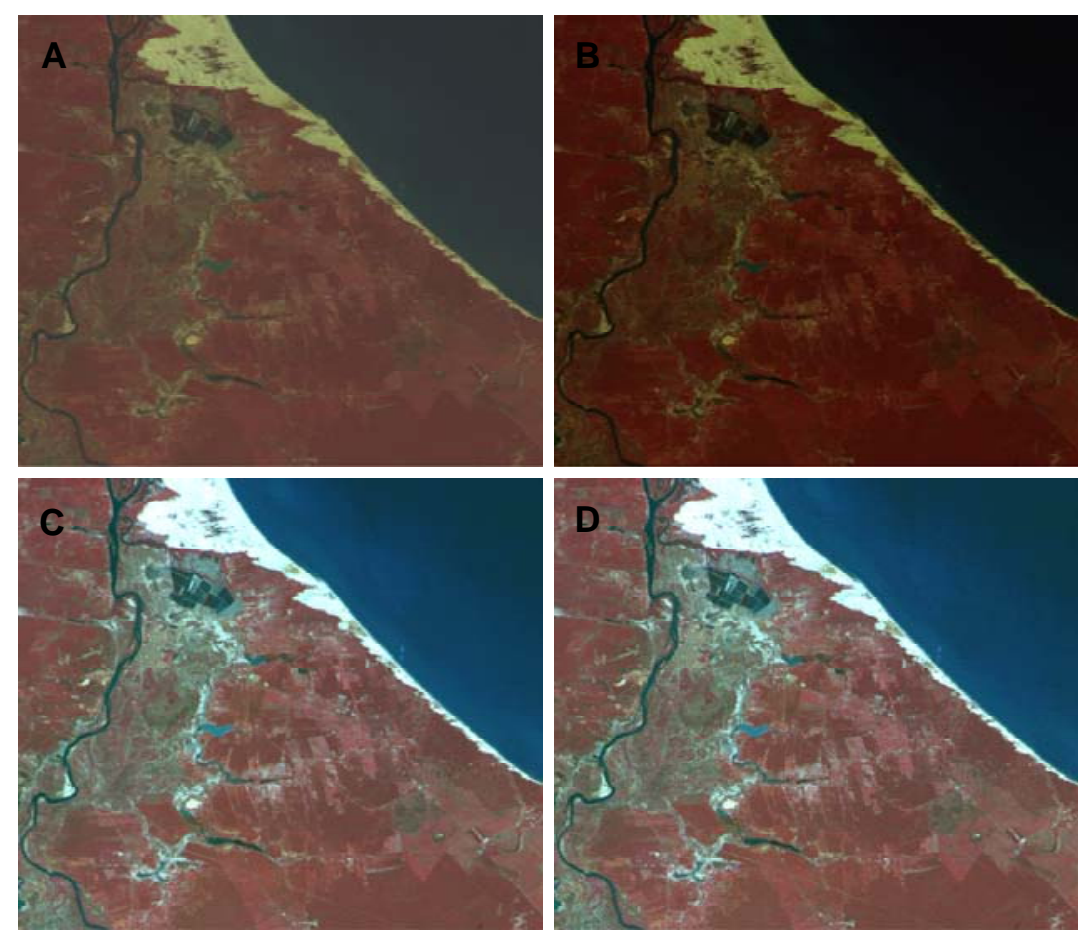

Figura 2. Imagens CCD/CBERS-1 em composição colorida (432). (A) imagem original; (B) imagem com correção atmosférica; (C) imagem após ajuste de média e variância; (D) imagem reamostrada. 


\subsection{Realce de Imagem}

Após a reamostragem dos pixels foi realizado o procedimento de fusão por IHS das bandas, que resultou em um aumento da nitidez e do detalhamento da imagem fusionada em comparação com a imagem reamostrada, representando um ganho de informação. Assim, a imagem que, anteriormente (Figura 3A), apresentava uma resolução espacial de 20 metros passou a apresentar uma resolução espacial equivalente a 5 metros (Figura 3B).
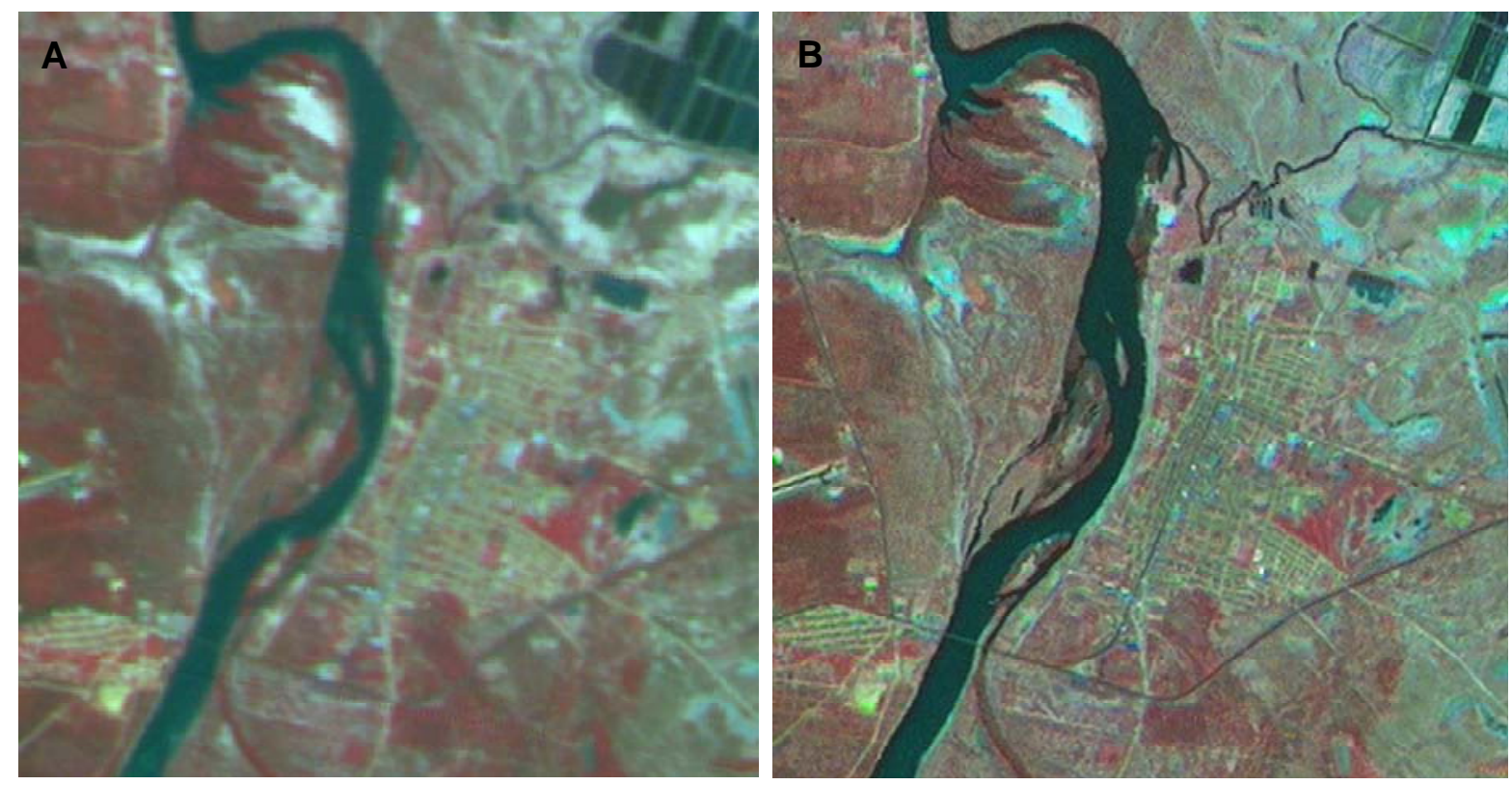

Figura 3. Imagens CCD/CBERS-1 em composição colorida (432) (recorte da área de estudo). (A) reamostrada; (B) resultado da fusão IHS das bandas 2,3 e 4 com a banda pancromática $\mathrm{ETM}^{+} /$Landsat-7.

Na imagem fusionada foi realizada a análise por Componentes Principais. O primeiro componente (PC-1) agrupou o maior número de informações (Tabela 1). Desta forma a segmentação e as classificações "pixel a pixel” foram realizadas a partir da imagem correspondente ao Primeiro Componente Principal (PC-1). Para realizar a segmentação da imagem foram feitos diversos testes, determinando-se os limiares de similaridade de 15 e área de 300 .

Tabela 1. Média, variância e contribuição de cada um dos Componentes Principais.

\begin{tabular}{c|rrrc}
\hline PC & Média & Variância & Auto-valor & $\begin{array}{c}\text { Porcentage } \\
\text { m }\end{array}$ \\
\hline PC-1 & 123,15 & 3391,75 & 3428,77 & 69,05 \\
PC-2 & 127,15 & 1462,73 & 1426,35 & 28,73 \\
PC-3 & 127,00 & 110,89 & 110,26 & 2,22 \\
\hline
\end{tabular}

\subsection{Avaliação das classificações}

\subsubsection{Porcentagem de acertos}

Considerando os algoritmos de classificação que necessitam de uma imagem segmentada, Isoseg e Bhattacharyya, observa-se que apesar do algoritmo Bhattacharyya ser um classificador supervisionado necessitando de amostras de treinamento e o Isoseg não 
necessitar desta etapa, os dois obtiveram resultados idênticos, coerentes com o tipo de informação presente na área de estudo (Figura 4A/B).

A matriz de erros obtida a partir desses classificadores revela que todas as classes representadas neste estudo foram subestimadas (erros de omissão), com exceção da classe Vegetação 2 e Duna que agruparam um número maior de classes que deveriam (erros de inclusão). Os classificadores não conseguiram identificar as classes Área Urbana e Manguezal (Tabela 2).

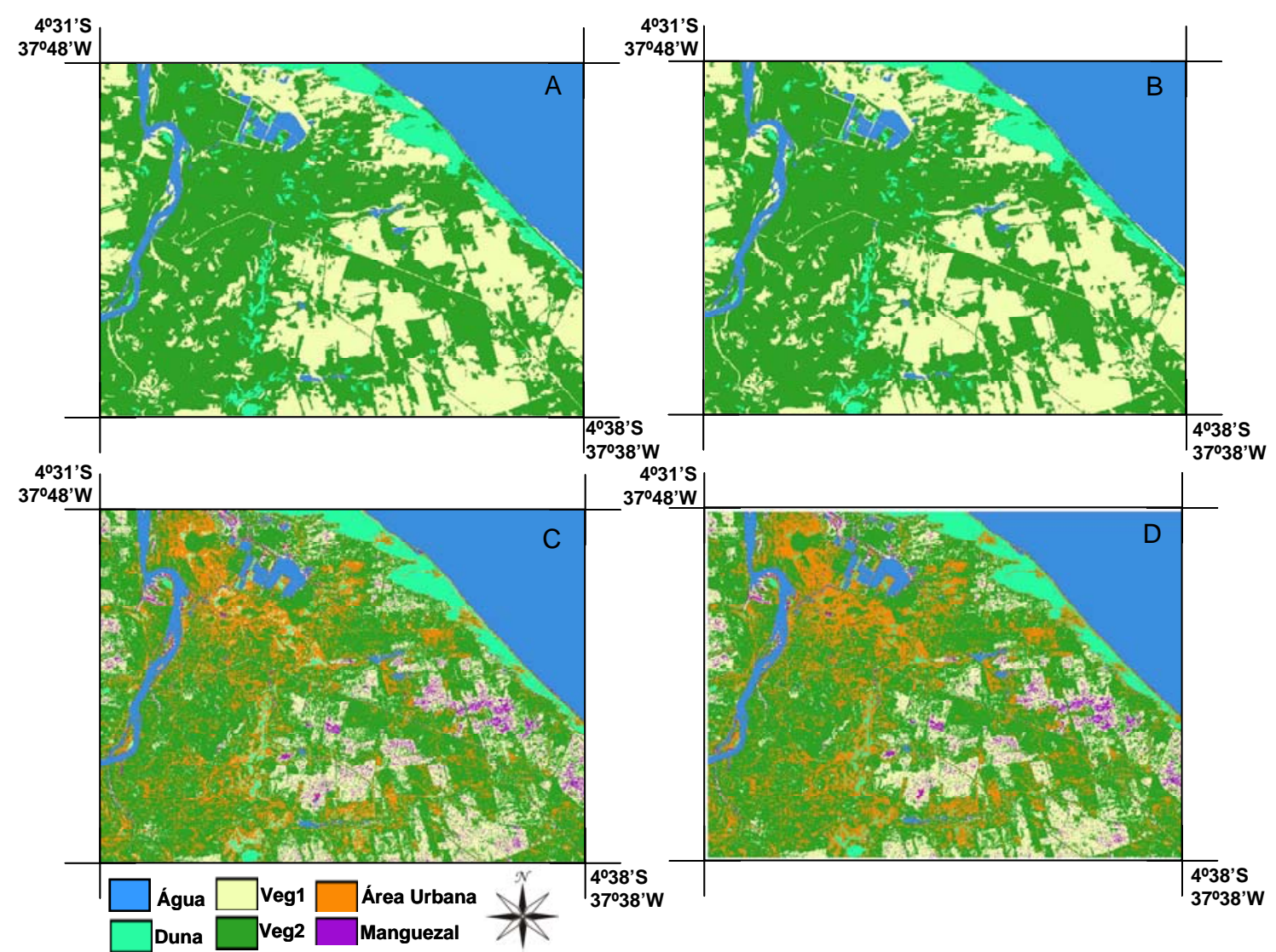

Figura 4. Classificação final das imagens CCD/CBERS-1 e ETM+/Landsat-7 a partir dos classificadores Isoseg (A), Bhattacharyya (B), Maxver (C) e Maxver-ICM (D).

Tabela 2. Matriz de erros (em porcentagem) dos classificadores Isoseg e Bhattacharyya.

\begin{tabular}{|c|c|c|c|c|c|c|c|}
\hline & Água & Veg. 1 & Veg. 2 & $\begin{array}{c}\text { Área } \\
\text { urbana }\end{array}$ & Manguezal & Duna & Total \\
\hline Água & 14,68 & 1,08 & 1,95 & 0 & 0 & 0,27 & 18,00 \\
\hline Vegetação 1 & 0,10 & 22,79 & 21,08 & 0 & 0 & 0,42 & 44,40 \\
\hline Vegetação 2 & 0,28 & 3,70 & 26,33 & 0 & 0 & 1,07 & 31,38 \\
\hline Área urbana & 0,09 & 0,04 & 1,41 & 0 & 0 & 0,01 & 1,54 \\
\hline Manguezal & 0,19 & 0,52 & 0,25 & 0 & 0 & 0,01 & 0,97 \\
\hline Duna & 0,00 & 0,16 & 0,52 & 0 & 0 & 3,03 & 3,72 \\
\hline Total & 15,35 & 28,30 & 51,54 & $\mathbf{0}$ & $\mathbf{0}$ & 4,80 & 100,0 \\
\hline
\end{tabular}

A partir dos resultados obtidos pelos classificadores "pixel a pixel” (Maxver e MaxverICM), podemos constatar que, visualmente, não houve grande diferença entre os dois métodos 
utilizados (Figura 4C/D). Esse resultado pode ser confirmado pela matriz de erros (Tabelas 3 e 4), em que se observa que esses dois algoritmos de classificação subestimaram as áreas ocupadas pelas classes Água e Vegetação 1 e superestimaram as demais classes.

Tabela 3. Matriz de erros (em porcentagem) do classificador Maxver.

\begin{tabular}{l|rrrrrrrr}
\hline & Água & Veg. 1 & Veg. 2 & $\begin{array}{c}\text { Área } \\
\text { urbana }\end{array}$ & Manguezal & Duna & Total \\
\hline Água & 14,84 & 0,49 & 1,49 & 0,69 & 0,27 & 0,22 & $\mathbf{1 8 , 0 0}$ \\
Vegetação 1 & 0,20 & 12,65 & 24,13 & 4,89 & 2,15 & 0,39 & $\mathbf{4 4 , 4 0}$ \\
Vegetação 2 & 0,37 & 1,90 & 20,04 & 8,11 & 0,24 & 0,72 & $\mathbf{3 1 , 3 8}$ \\
Área urbana & 0,09 & 0,06 & 0,85 & 0,51 & 0,01 & 0,02 & $\mathbf{1 , 5 4}$ \\
Manguezal & 0,26 & 0,23 & 0,26 & 0,07 & 0,15 & 0,01 & $\mathbf{0 , 9 7}$ \\
Duna & 0,01 & 0,18 & 0,32 & 0,32 & 0,01 & 2,86 & $\mathbf{3 , 7 2}$ \\
\hline Total & $\mathbf{1 5 , 7 6}$ & $\mathbf{1 5 , 5 2}$ & $\mathbf{4 7 , 1 0}$ & $\mathbf{1 4 , 5 9}$ & $\mathbf{2 , 8 2}$ & $\mathbf{4 , 2 1}$ & $\mathbf{1 0 0 , 0}$
\end{tabular}

Tabela 4. Matriz de erros (em porcentagem) do classificador Maxver-ICM.

\begin{tabular}{l|crrrrrrr}
\hline & Água & Veg. 1 & Veg. 2 & \multicolumn{2}{c}{$\begin{array}{c}\text { Área } \\
\text { urbana }\end{array}$} & Manguezal Duna & Total \\
\hline Água & 14,86 & 0,35 & 1,48 & 0,81 & 0,26 & 0,23 & $\mathbf{1 8 , 0 0}$ \\
Vegetação 1 & 0,19 & 12,63 & 24,16 & 5,18 & 1,81 & 0,42 & $\mathbf{4 4 , 4 0}$ \\
Vegetação 2 & 0,37 & 1,12 & 20,15 & 8,72 & 0,22 & 0,81 & $\mathbf{3 1 , 3 8}$ \\
Área urbana & 0,09 & 0,02 & 0,85 & 0,55 & 0,01 & 0,02 & $\mathbf{1 , 5 4}$ \\
Manguezal & 0,26 & 0,22 & 0,26 & 0,07 & 0,15 & 0,01 & $\mathbf{0 , 9 7}$ \\
Duna & 0,01 & 0,06 & 0,3 & 0,44 & 0,01 & 2,88 & $\mathbf{3 , 7 2}$ \\
\hline Total & $\mathbf{1 5 , 7 9}$ & $\mathbf{1 4 , 4}$ & $\mathbf{4 7 , 2 0}$ & $\mathbf{1 5 , 7 8}$ & $\mathbf{2 , 4 7}$ & $\mathbf{4 , 3 6}$ & $\mathbf{1 0 0 , 0}$
\end{tabular}

\subsubsection{Cálculo de área}

As áreas correspondentes a cada classe foram comparadas com as áreas das classes do mapa temático utilizado como verdade de campo. Todos os classificadores subestimaram as classes Água e Vegetação 1 e superestimaram a classe Vegetação 2. Os classificadores Isoseg e Bhattacharyya não conseguiram classificar a classe Área Urbana e Manguezal e os classificadores Maxver e Maxver-ICM superestimaram a área dessas duas classes. A classe Dunas foi superestimada por todos os classificadores utilizados (Tabela 5).

\subsubsection{Coeficiente Kappa}

Com base no coeficiente Kappa calculado, percebe-se que dentre todas as classes, a classe Duna foi melhor classificada por Isoseg e Bhattacharyya e a classe Água por Maxver e Maxver-ICM. As demais classes não obtiveram uma classificação muito boa, variando de 66 a $0 \%$ de acurácia (Tabela 6). Essas duas classes (Duna e Água) apresentam um comportamento espectral bastante distinto das demais, sendo facilmente diferenciadas e classificadas. Apesar das três classes de vegetação também apresentarem um comportamento espectral diferenciado, houve confusão na sua classificação, o que resultou num baixo coeficiente Kappa. Da mesma forma, as classes Área Urbana e Manguezal foram mal classificadas, obtendo-se um baixo índice Kappa. 
GÓES, C. A.; MELLO FILHO W. L.; CARVALHO, M. Avaliação do desempenho de diferentes classificadores

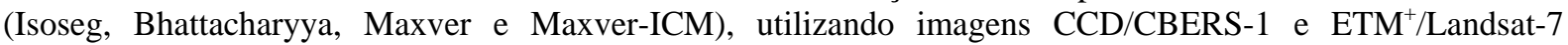
fusionadas. Ambi-Agua, Taubaté, v. 1, n. 2, p. 80-89, 2006. (doi:10.4136/ambi-agua.14)

Tabela 5. Matriz da área de cada classe $\left(\mathrm{km}^{2}\right)$.

\begin{tabular}{l|c|rrrrr}
\hline Classes\Classificador & Verdade de campo & Isoseg & Bhattacharyya & Maxver & & Maxver- ICM \\
\cline { 2 - 5 } & 47,03 & 40,11 & 40,12 & 41,195 & 41,27 \\
Água & 116,03 & 73,97 & 73,971 & 40,55 & 37,63 \\
Vegetação 1 & 82,01 & 134,70 & 134,70 & 123,09 & 123,36 \\
Vegetação 2 & 4,03 & 0 & 0 & 38,14 & 41,24 \\
Área urbana & 2,53 & 0 & 0 & 7,38 & 6,44 \\
Manguezal & 9,71 & 12,55 & 12,55 & 11,00 & 11,41 \\
Duna & $\mathbf{2 6 1 , 3 5}$ & $\mathbf{2 6 1 , 3 5}$ & $\mathbf{2 6 1 , 3 5}$ & $\mathbf{2 6 1 , 3 5}$ & $\mathbf{2 6 1 , 3 5}$
\end{tabular}

Tabela 6. Coeficiente Kappa de concordância entre a imagem classificada e a verdade de campo.

\begin{tabular}{l|ccccc}
\hline Classes\Classificador & Isoseg (95\%) & $\begin{array}{c}\text { Bhattacharyya } \\
\mathbf{( 9 5 \% )}\end{array}$ & Maxver (95\%) & $\begin{array}{c}\text { Maxver- } \\
\text { ICM (95\%) }\end{array}$ \\
\hline Água & 0,7826 & 0,7826 & 0,7917 & 0,7932 \\
Vegetação1 & 0,3213 & 0,3213 & 0,1537 & 0,1641 \\
Vegetação2 & 0,6677 & & 0,6677 & 0,3169 & 0,3220 \\
Área urbana & 0,0000 & & 0,0000 & 0,2201 & 0,2375 \\
Manguezal & 0,0000 & & 0,0000 & 0,1257 & 0,1290 \\
Duna & 0,8065 & 0,8065 & 0,7598 & 0,7655 \\
\hline Total & $\mathbf{0 , 5 1 4 6}$ (reg.) & $\mathbf{0 , 5 1 4 6}$ & $\mathbf{0 , 3 4 8 1}$ (ruim) & $\mathbf{0 , 3 5 4 1}$
\end{tabular}

Dentre os quatro classificadores testados, os que apresentaram melhores resultados foram os que utilizam uma imagem segmentada para realizar a classificação (Isoseg e Bhattacharyya). O delineamento prévio das classes espectrais semelhantes a partir da segmentação mostrou ser bastante apropriado para se obter uma boa classificação e, apesar do algoritmo utilizado pelo classificador Bhattacharyya necessitar de amostras de treinamento para realizar a classificação, o resultado foi idêntico ao obtido pelo classificador Isoseg, que não necessita de amostras de treinamento. Dessa forma, o classificador Isoseg pode ser utilizado com maior facilidade, dispensando o conhecimento prévio da área de estudo.

As baixas concordâncias obtidas pelos diferentes métodos de avaliação utilizados neste estudo refletem a confusão entre as diferentes classes, que pode ter ocorrido devido ao desempenho dos classificadores, mas que foi influenciada principalmente pela qualidade da imagem, que apesar de ter passado por um procedimento de pré-processamento não apresentou condições satisfatórias para distinguir as classes analisadas.

Apesar da reamostragem dos pixels ter melhorado a resolução espacial da imagem, definindo melhor os alvos de interesse, a resolução espectral pode ter sido comprometida tanto na aquisição da imagem quanto no seu processamento, resultando nos baixos índices de concordância encontrados.

Deve ser levado em consideração que, apesar da área não apresentar um dinamismo muito alto, as datas das imagens não são coincidentes e houve uma edição matricial do mapa temático que foi utilizado como verdade de campo, o que pode ter afetado o resultado comparativo do experimento. 


\section{CONCLUSÕES}

Os classificadores que apresentaram o melhor desempenho para a área de estudo, discriminando os diferentes tipos de alvos, foram aqueles que utilizam uma imagem segmentada (Isoseg e Bhattacharyya). Dentre esses dois classificadores, o Isoseg pode ser considerado como o mais eficiente, por apresentar uma classificação coerente com a verdade de campo e dispensar o conhecimento prévio da área de estudo, não necessitando de amostras de treinamento. A dificuldade encontrada nesse tipo de classificação é a obtenção da imagem segmentada, uma vez que devem ser testados diversos limiares de segmentação até se obter uma segmentação satisfatória, e esse processo influenciará diretamente a qualidade final da classificação.

A avaliação das classificações deve ser realizada por diferentes métodos e recomenda-se que sempre seja apresentada a matriz de porcentagem de acertos (matriz de erros) em cada mapa de uso e cobertura do solo produzido, dessa forma o usuário tem a possibilidade de selecionar a melhor classificação de acordo com a sua necessidade, levando em consideração a importância relativa das classes de interesse. Neste estudo, além da porcentagem de acertos, foram utilizados outros dois procedimentos de análise dos resultados: cálculo de área e índice Kappa. O primeiro possibilitou observações quantitativas sobre as classificações e o segundo permitiu analisar o grau de concordância entre as imagens classificadas e a verdade terrestre. Dessa forma, a escolha desses três procedimentos de análise foram complementares, permitindo uma avaliação mais precisa e completa dos mapas temáticos produzidos.

O procedimento de fusão da imagem pela transformação IHS possibilitou uma melhora na resolução espacial, auxiliando na distinção dos diferentes alvos e garantindo uma boa classificação, porém a informação espectral das imagens é imprescindível para garantir a completa qualidade da classificação.

\section{REFERÊNCIAS BIBLIOGRÁFICAS}

BARRETT, E. C.; CURTIS, L. F. Introduction to environmental remote sensing. 3 ed. London: Chapman and Hall, 1992. 426 p.

CAMARA, G.; SOUZA, R. C. M.; FREITAS, U. M.; GARRIDO, J. SPRING: Integrating remote sensing and GIS by object-oriented data modelling. Computers \& Graphics, [S.l.], v. 20, n. 3, p. 395-403, 1996.

CARVALHO, M. Mapeamento da sensibilidade ao impacto por óleo de um segmento da costa entre os Estados do Rio Grande do Norte e Ceará utilizando imagens ETM+/Landsat-7 e geoprocessamento. 279f. 2003. Dissertação (Mestrado em Sensoriamento Remoto) - Instituto Nacional de Pesquisas Espaciais, São José dos Campos, 2003.

CHAVEZ JR., P. S. An improved dark-object subtraction technique for atmospheric scattering correction of multispectral data. Remote Sensing of Environment, New York, v. 24, p. 459-479, 1988.

COHEN, J. A. A coefficient of agreement for nominal scales. Educational and Psychological Measurement, Thousand Oaks, v. 20, n. 1, p.37-46, 1960. 
CONGALTON, R. G.; ODERWALD, R. O.; MEAD, R. A. Assessing Landsat classification accuracy using discrete multivariate analysis statistical techniques. Photogrammetric Engineering and Remote Sensing, Bethesda, v. 49, n. 12, p.1671-1678, 1983.

FEDOROV, D.; FONSECA, L. M. G. Restau: image restoration 0.0.15. São José dos Campos: INPE, Divisão de Processamento de Imagens, 2002

FOODY, G. M. On the compensation for chance agreement in image classification accuracy assessment. Photogrammetric Engineering and Remote Sensing, Bethesda, v. 58, n. 10, p.1459-1460,1992

INSTITUTO NACIONAL DE PESQUISAS ESPACIAIS. SPRING: sistema de processamento de informações georreferenciadas: manual de ajuda. São José dos Campos: INPE, 2004.

LILLESAND, T. M.; KIEFER, R. W.; CHIPMAN J. W. Remote sensing and image interpretation. 5 ed. Chichester: John Wiley \& Sons, 2004. 763 p.

MATHER, P. M. Computer processing of remotely-sensed images: an introduction. 3 ed. Chichester: John Wiley \& Sons, 2004. 324 p.

RICHARDS, J. A.; JIA, X. Remote sensing digital image analysis: an introduction. 4 ed. Berlin: Springer-Verlag, 2006. 439 p.

ROSENFIELD, G. H. Analysis of thematic map classification error matrices. Photogrammetric Engineering and Remote Sensing, Bethesda, v. 52, n. 5, p. 681686, 1986.

ROSENFIELD, G. H.; FITZPATRICK-LINS, K. A coefficient of agreement as a measure of thematic classification accuracy. Photogrammetric Engineering and Remote Sensing, Bethesda, v. 52, n. 2, p. 223-227, 1986. 